\title{
Triathlon transition study: quantifying differences in running movement pattern and precision after bike-run transition
}

\author{
Christian Weich $^{\mathrm{a}}$ (D), Randall L. Jensen ${ }^{\mathrm{b}}$ and Manfred Vieten ${ }^{\mathrm{c}}$ \\ ${ }^{a}$ Department of Sports Science, University of Constance, Constance, Germany; ${ }^{b}$ Department of Health, Physical \\ Education \& Recreation, Northern Michigan University, Marquette, MI, USA; 'Department of Sports Science, \\ University of Constance, Constance, Germany
}

\begin{abstract}
Various publications discuss the discrepancies of running in triathlons and stand-alone runs. However, those methods, such as analysing step-characteristics or ground-contact time, lack the ability to quantitatively discriminate between subtle running differences. The attractor method can be applied to overcome those shortcomings. The purpose was to detect differences in athletes' running patterns $(\delta M)$ and movement precision $(\delta D)$ by comparing a 5,000 m run after a prior cycling session (TRun) with an isolated run over the same distance (IRun). Participants completed the conditions on a track and a stationary trainer, allowing the use of their personal bike to simulate an Olympic triathlon. During each run, three-dimensional acceleration data, using sensors attached to the ankles, were collected. Results showed that both conditions lead to elevated attractor parameters $(\delta M$ and $\delta D$ ) over the initial five minutes before the athletes found their rhythm. This generates a new perspective because independent of running after a bike session or without preload, an athlete needs certain time to adjust to the running movement. Coaches must consider this factor as another tool to fine-tune pacing and performance. Moreover, the attractor method is a novel approach to gain deeper insight into human cyclic motions in athletic contexts.
\end{abstract}

\section{KEYWORDS}

Attractor method; human cyclic motion; running quality; triathlon

\section{Introduction}

Triathlon is an endurance sport consisting of the three fundamental sport disciplines: swimming, cycling and running in a consecutive order. It is vital to note that competing in a triathlon event is more than just the summation of the mentioned three parts. Rather it develops its own dynamic as each discipline depends on the one performed before it, which leads to a much more challenging running portion compared to a stand-alone run. Wellington (2013), one of the most successful female long-distance triathletes ever, described the bikerun transition as a strange sensation of having legs like jelly. The duration and handling of this crucial transition phase can decide if an athlete finishes with a gold or a silver medal; a crucial performance factor observed in the 2012 London Olympic final (Stafford, 2012) 
between Sweden's Lisa Norden and the Swiss Nicola Spirig. The ability to cope with running after the cycle-run transition is likely the most crucial variable for success in triathlon competitions, especially in short-distance events (Chapman et al., 2009; Vleck, Burgi, \& Bentley, 2006). Many interesting attempts have been published to show the differences between competing in a triathlon in contrast to starting in a single running event. It appears that running movement patterns of triathletes are influenced by prior cycling in a way that the running motion becomes less efficient. Since the early 1990s, scientists and coaches within the field have been investigating and trying to minimise this interference (Gohlitz, Große, \& Witt, 1994; Witt, 1994). Most have examined this with conventional parameters like stride frequency or stride length and ground-contact time. Recently, EMG applications (Walsh, Stamenkovic, Lepers, Peoples, \& Stapley, 2015) have been available more easily (lower asset cost, technical enhancements). Hence, they have opened new opportunities, in particular to examine muscular activation and sensomotoric control during human gait. These and some further measurements were generally complemented by cardiovascular or respiratory data, such as heart rate, breathing analysis and lactate, and emotional values, such as the expression of athletes' perceived exertion (RPE) by Borg (1998). All these data parameters were also used to explain and enhance the process of transition in duathlon and triathlon events. The main outcome of these studies was the training method called 'brick workout' (Friel \& Vance, 2013, p. 441; Walsh, Hohl, Strang, \& Haworth, 2014), a session where the athletes combine the different disciplines one after the other, mainly focusing on cycling and running. To improve and develop the quantification of cyclic motion (as running, walking, swimming, cycling, etc.), especially human gait and the walking movement, we introduced a new approach in the field of cyclic motion analysis named 'attractor method' (Vieten, Sehle, \& Jensen, 2013; Vieten \& Jensen, 2015). In the last decades, many scientists calculated the maximal Lyapunov exponent (Alligood, Sauer, \& Yorke, 1997; Lyapunov, 1992) using Taken's embedding theorem (Perc, 2005; Taken, 1981). These are nonlinear approaches to judge the stability, providing results on human locomotion at group level but are not sensitive enough to analyse individuals' changes. This is where the attractor method initiates the possibility of using movement data sets containing fluctuations that result in stochastic changes, which are also applicable to the individual case. To get the desired insight into the very complex mechanisms of human motion, the attractor method was developed and firstly used in clinical settings dealing with multiple sclerosis patients at the Clinic Schmieder in Konstanz, Germany (Sehle, Vieten, Sailer, Muendermann, \& Dettmers, 2014). The rationale to apply this method in the context of competitive sports is based on promising results of initial studies yielding very sensitive results allowing the analysis of subtle changes in movement patterns. The objective of this investigation is to find out more about differences of an Isolated Run (IRun) and running after transiting from biking to running (Transition Run-(TRun)). This study is a first approach trying to find an appliance suitable for competitive athletes and trainers to evaluate and eventually improve individual running behaviour. In addition, it may enhance overall performance, such as shortening their cycling-affected running time in triathlon events. The analysis focused on the resulting attractors based on triaxial accelerometer data collected during the runs.

Hence, we aimed to quantify relevant differences in all participants' running movement pattern $(\delta M)$ and in movement precision $(\delta D)$ after undergoing a cycling session before running 5,000 $\mathrm{m}$ compared to a single run over the same distance. It was hypothesised that the IRun would develop without notable fluctuations for the calculated parameters ( $\delta M$ and 
$\delta D)$ over the whole course of time, whereas we expected that during the TRun, the cycling involvement would cause divergent values at the onset of the run.

\section{Methods}

\section{Participants}

A total of 36 participants were tested from January until June 2016 in Marquette, Michigan, USA, and Konstanz, Germany. Two participants were excluded from further analysis caused by the following reasons: one participant dropped out after the first test, and another one was not able to fulfil the requirements of the study. Thirty-four athletes, ten females and 24 males, participated in the study. The mean \pm SD age, height and body mass were $26.64 \pm 6.86$ years, $175.0 \pm 9.5 \mathrm{~cm}$ and $69.47 \pm 11.64 \mathrm{~kg}$. The participants were active triathletes $(n=13)$, runners $(n=12)$ or sportspersons performing other kinds of sports, containing no endurance specific running parts $(n=9)$, e.g., weightlifting or climbing. Their scope of training aggregated to $10.1 \pm 5.9 \mathrm{~h}$ weekly, and none had suffered any present injury, which could have possibly impeded their performance. The only prerequisites were to be aged between 18 and 45 years and that everyone should be able to run ten kilometres below $50 \mathrm{~min}$ (equal to a 5:00 min per kilometre pace). All participants were requested to fill out and sign an informed consent and a questionnaire checking their health status and physical activity (Thomas, Reading, \& Shepard, 1992).

\section{Equipment}

To collect the necessary raw accelerometer data, two inertial sensors were used (RehaWatch by Hasomed, Magdeburg, Germany) which were attached to both ankles by a hook-and-loop fastener. The sensors have a size of $60 \times 35 \times 15 \mathrm{~mm}$ and weight $35 \mathrm{~g}$ each. They function as a triaxial accelerometer with up to $16 \mathrm{G}$, a triaxial gyroscope with up to $2,000 \% \mathrm{~s}$ and magnetometer measuring with 1.3 Gaus. The possible measuring rate is up to $600 \mathrm{~Hz}$, and they are constructed as a microelectromechanical system (MEMS).

They measured the acceleration of the feet in three dimensions $(x, y, z)$. In addition, a Lactate Scout (SensLab, Leipzig, Germany) and a heart rate monitor (Polar, Kempele, Finland) were used to determine the individual anaerobic threshold (IAT) (Coen, Urhausen, \& Kindermann, 2001; Stegmann, Kindermann, \& Schnabel, 1981). The cycling tests were conducted on a cycling trainer (Fortius Multiplayer by Tacx, Wassenaar, the Netherlands) or Lode Excalibur (Groningen, the Netherlands), and the running speed was paced using an ad hoc engineered pacing system called Running Rabbit (in-house development). ${ }^{1}$

\section{Experimental protocol and data measurement}

The participating athletes repeated the testing protocol in a time frame of approximately ten days consisting of three testing days (Figure 1). The initial sessions were conducted to determine the IAT for both disciplines, cycling and running, which led to the necessary power and pace values to ensure an individual-specific control. With this information, the workloads for the sessions on days two and three were determined. Both conditions were performed in a random order by each participant. When participants finally arrived for 


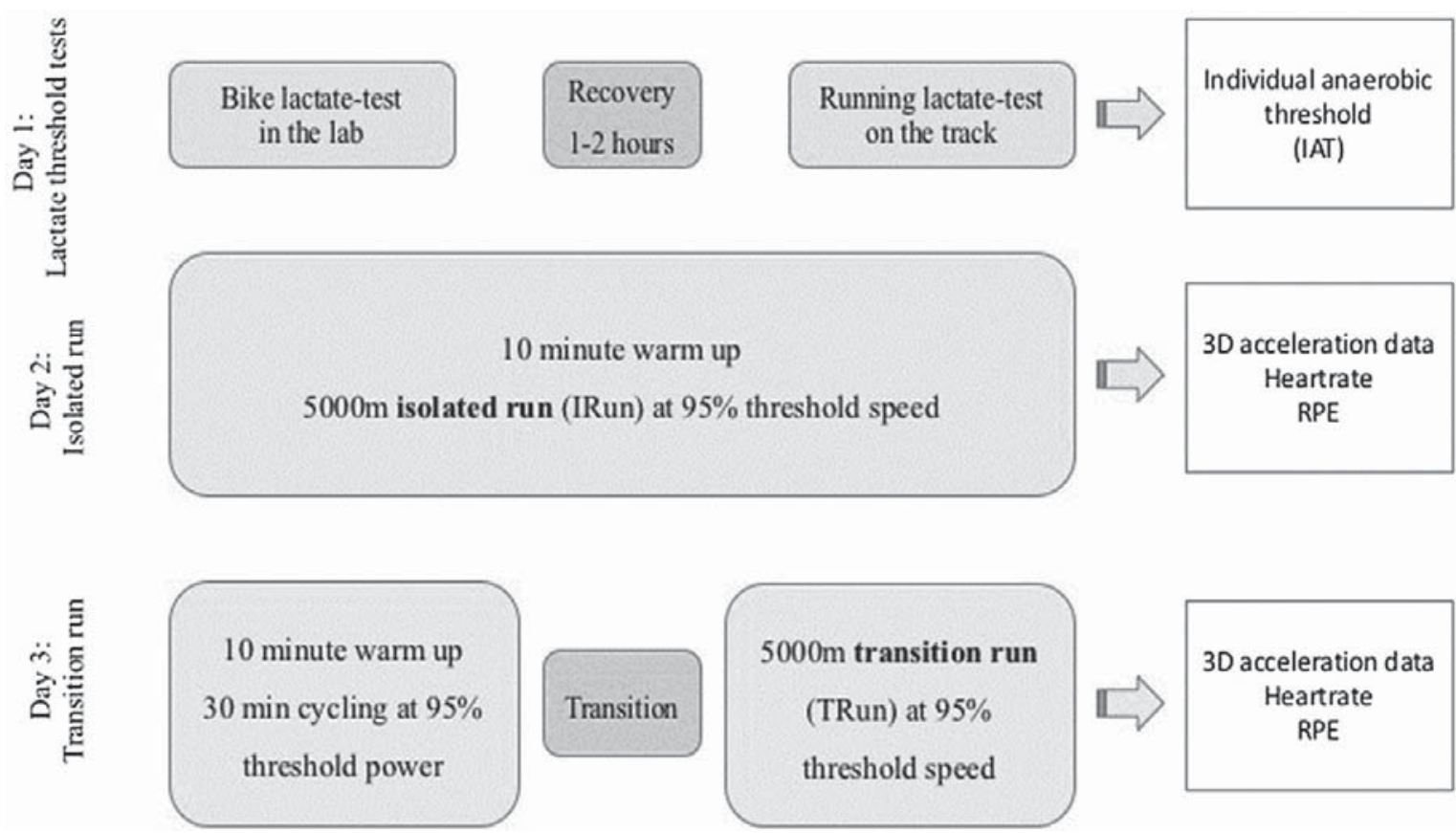

Figure 1. Overview of the three testing days and the measured output values (RPE: Rate of perceived exertion).

the 5,000 m tests, they were equipped with a heart rate strap around the chest and with two acceleration sensors attached to the ankles above each lateral malleolus. Test day 2 consisted of an isolated $5,000 \mathrm{~m}$ run on a $200 \mathrm{~m}$ or a $400 \mathrm{~m}$ track at $95 \%$ IAT pace to simulate the intensity of an Olympic triathlon (Friel \& Vance, 2013, p. 399). It was preceded by a ten-minute warm-up where the participants ran 1,200 m self-selected speed with two accelerations over $25 \mathrm{~m}$ during the last $400 \mathrm{~m}$. The warm-up was completed with 5-min running at goal pace of the actual 5,000 $\mathrm{m}$ run, which immediately followed the warm-up. Between the warm-up phase and the actual run, the athlete was instructed to start the sensors at his ankle and simultaneously a person with a bike, data receiver and laptop joined in to collect the raw data.

At least $48 \mathrm{~h}$ later, they performed the same procedure again but this time they warmed up for ten minutes on a stationary bike including two minutes of their goal wattage (there was no warming up for the ensuing running portion). This protocol was followed by a 30-min cycling session at approximately $95 \%$ of their IAT wattage to simulate an Olympic triathlon bike split (Friel \& Vance, 2013, p. 399). When they finished cycling, they changed to their running shoes, all necessary devices described above were switched on, and the transition to the run started. As soon as they arrived at the first light of the pacing system, the $5,000 \mathrm{~m}$ transition run began similar to the 5,000 m isolated run. All runs were paced with the Running Rabbit pacing system to assure a constant and comparable running velocity of $95 \%$ IAT. To collect the data, the athlete was, as with the IRun, accompanied by a cyclist transporting a laptop with a Bluetooth receiver. These data were received from triaxial accelerometers at a sampling frequency of $300 \mathrm{~Hz}$. The raw data were assembled in one-minute intervals during the IRun and TRun, respectively, starting with the initial measurement from the beginning of the actual run until $60 \mathrm{~s}$ had elapsed. Data were collected on alternate minutes beginning with minute $1,3,5$, etc., until the participants finished the 5,000 $\mathrm{m}$. For all data collections, acceleration data were chosen. Alternative data acquisition would have 
been a 'kinematic tracking system [which] requires large degree of experimental effort and expense' (Vieten et al., 2013, p. 3). Furthermore, the athletes were asked to report their subjective exertion every kilometre on a 1-10 scale (Borg, 1998).

\section{Data analysis}

The software Statfree (Vieten Dynamics, Germany) was used to make the raw data comparable for both trials. A raw data file contains seven columns: time and the acceleration in $x, y$ and $z$ direction for the left and the right foot, respectively. These raw data should consequently be transferred to objective numbers to quantify the differences in human gait data. Based on the calculation of the attractor of each measuring interval (1) and its fluctuations (2), the main output terms ${ }^{2}$ are $\delta M$, the velocity normalised difference between two attractors, for instance, between two running intervals (3), and $\delta D$ representing the difference between the variability of two compared attractors' fluctuations (4) (Vieten et al., 2013, p. 3).

$$
\begin{gathered}
\vec{A}_{a, c}\left(\tau_{j}\right)=\frac{1}{n} \sum_{i=1}^{n} \vec{a}_{a, C}\left(i \cdot \tau_{j}\right)+\frac{1}{n} \sum_{i=1}^{n} \vec{b}_{a, C}\left(t=i \cdot \tau_{j}\right) \approx \frac{1}{n} \sum_{i=1}^{n} \vec{a}_{a, C}\left(i \cdot \tau_{j}\right) \\
D_{a, C}\left(\tau_{j}\right)=\sqrt{\frac{1}{n-1}} \sum_{i=1}^{n}\left[\vec{A}_{a, C}\left(\tau_{j}\right)-\vec{a}_{a, C}\left(i \cdot \tau_{j}\right)\right]^{2}
\end{gathered}
$$

where $t$ is the time, $a$ stands for right or left foot and $C$ represents two different time intervals of the compared run.

$\delta M$ is the parameter containing the information about changes in the individual running pattern or style,

$$
\delta M=\frac{1}{v} \sqrt{\sum_{i=1}^{3}\left[\left\langle\left(A_{r, B, x_{i}}-A_{r, E, x_{i}}\right)^{2}\right\rangle+\left\langle\left(A_{l, B, x_{i}}-A_{l, E, x_{i}}\right)^{2}\right\rangle\right]}
$$

where $v$ is the running speed; $r$ and $l$, right and left foot; $B=$ begin and $E=$ end denote two different time intervals; and $\langle\ldots\rangle$ means the average of the included expression.

Secondly, $\delta D$ informs about movement precision, i.e., the way how smooth a person runs.

$$
\delta D=\sqrt{\left\langle\left(D_{r, B}-D_{r, E}\right)^{2}\right\rangle+\left\langle\left(D_{l, B}-D_{l, E}\right)^{2}\right\rangle}
$$

$\delta M$ and $\delta D$ are abstract expressions, and the dimensions are not of particular importance. Nevertheless, $\delta M$ is given in s, while $\delta D$ and absolute $D$ have the dimension $\mathrm{m} / \mathrm{s}^{2}$.

Moreover, an averaged attractor (AA) was calculated for each participant's IRun and TRun including the means of all attractors from interval minute 7-17. The AA contained the middle part of each run where the athlete had already found his or her running rhythm, but had not accumulated too much fatigue. 


\section{General information}

Except for the five fastest athletes, everyone else ran the $5,000 \mathrm{~m}$ in at least $21 \mathrm{~min}$ so that this interval was set as the final one. For all athletes who ran only 19 min over the $5 \mathrm{~km}$, interval minute 19 counted as the last measuring and accordingly minute 21 did not contain any value. Furthermore, in some cases, a faulty Bluetooth connection between the runners' sensors and the collecting device caused data gaps. To fill these gaps, we applied a spline approximation (using Statfree, Vieten Dynamics, Germany) for gaps small enough to be in accordance with the sampling theorem (Shannon, 1948, 1949).

\section{Individual-Run-Ratio (IRR)}

To get the time course of the data representing the running style $(\delta M)$ and the movement precision $(\delta D)$, each time interval (minutes 1-21) of both runs was compared with the AA of the respective run (Figure $2(\mathrm{~A})$ ). As a result, $\delta M$ and $\delta D$ data values were obtained for each person and run, which were transferred to SPSS (IBM, Armonk, USA, version 20.0.0) for further statistical analysis.

\section{Stable running section (SS)}

Analogue to the determination of the $A A$ within one person's run, the central part was chosen, i.e., the $\delta M$ and $\delta D$ outcomes from minute 7 to 17 (vs. AA), which represent a section where the participants found their running rhythm, and fatigue is not yet a critical performance-inhibiting factor. By this means, the stable section (SS, Figure 2(B)) could
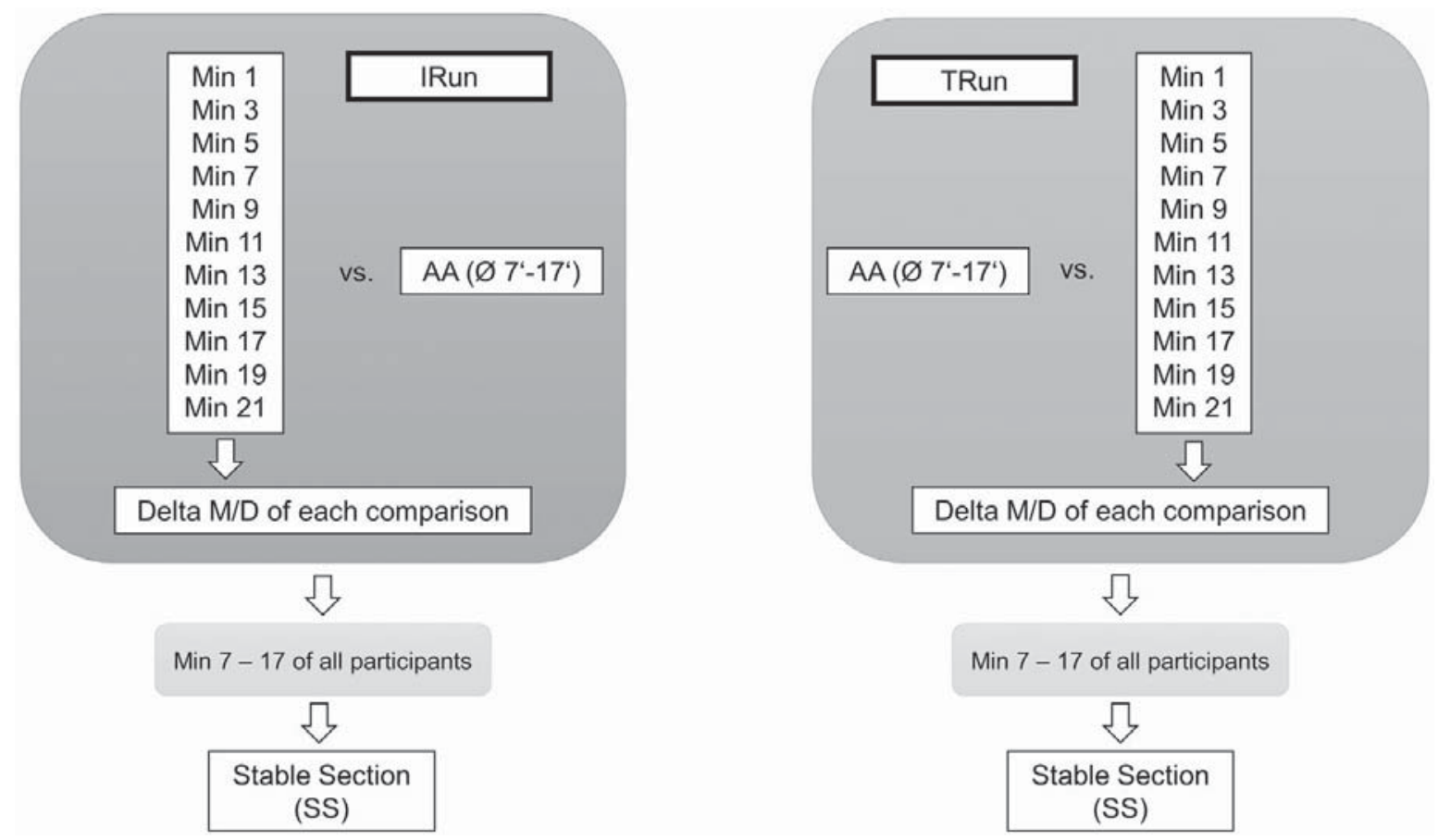

Figure 2. Model of the Individual-Run-Ratio (IRR, A) creating the data to be tested. AA was calculated as the averaged attractor from minutes $7-17$. And the resulting stable sections (SS, B) resulting from the $\delta M$ and $\delta D$ values from minutes $7-17$ of all participants. 
be determined across all participants. Therefore, two new variables were created (SPSS) containing the mean of all participants $\delta$-values between minute 7 and 17 .

\section{Overall-Run-Ratio (ORR)}

Finally, to test the hypothesis about the development of both conditions, an isolated run compared to the run with a prior bike session (transition run), paired $t$-tests were performed using SPSS (ORR, Figure 3). Because the current study focused mainly on the initial and the final parts of both runs (the middle section was stable), the single $\delta M$ s and $\delta D$ s from minute 1, 3 and 5 of each run were paired and contrasted against SS. The same was applied for the final two measurements, minute 19 and 21. Data for $\delta M$ and $\delta D$ were assessed for normality and found to display a normal distribution. Furthermore, there was no order effect for testing detected.

\section{Results}

Both running forms, IRun and TRun, showed highly significant $(p<0.001)$ differences for the initial minute (= onset of run) compared to the stable section. This finding prevails for the running pattern $\delta M$ and as well for athletes' running variability $\delta D$ (see Figures 4 and 5).

Furthermore, the $\delta M$ of minutes three and five for both runs was also significantly different from SS ( $p=0.001$ for min 3 in both runs, $p=0.004$ for the IRun and $p=0.044$ for the TRun). In contrast to the start of the runs, the final two measurement intervals (minutes $19-21)$ were not statistically different from SS (for $\delta D_{\text {IRun }} p=0.089(\min 19), p=0.101(\mathrm{~min}$ $21), \delta D_{\text {TRun }} p=0.249(\min 19), p=0.052(\min 21), \delta M_{\text {IRun }} p=0.095(\min 19)$ and $\delta M_{\text {TRun }}$ $p=0.059(\min 19), p=0.079(\min 21))$ except $\delta M_{\text {IRun }}$ at minute 21 vs. the mean $p=0.033$.

\section{Discussion and implications}

The main outcome of the results indicates that both running conditions, in terms of the running pattern and the variability, behave similarly, regardless if athletes just run 5,000 m at a given velocity (here around anaerobic threshold) or run after a prior bike session. Before the athletes finally found their rhythm, they passed through a phase with a slightly deviating individual running pattern in combination with a departure of movement precision. The latter has often been reported empirically as a, if only marginal, feeling of shaky stride performance and suboptimal body position (Wellington, 2013). Although these outcomes
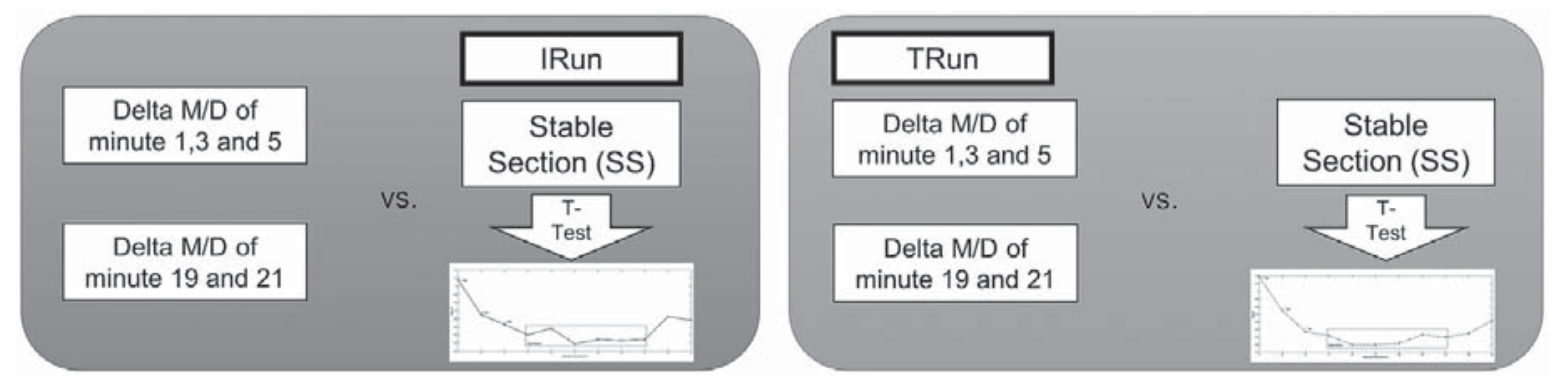

Figure 3. Model of the Overall-Run-Ratio (ORR) comparing onset and end of each running condition with the stable part to get the final results. 


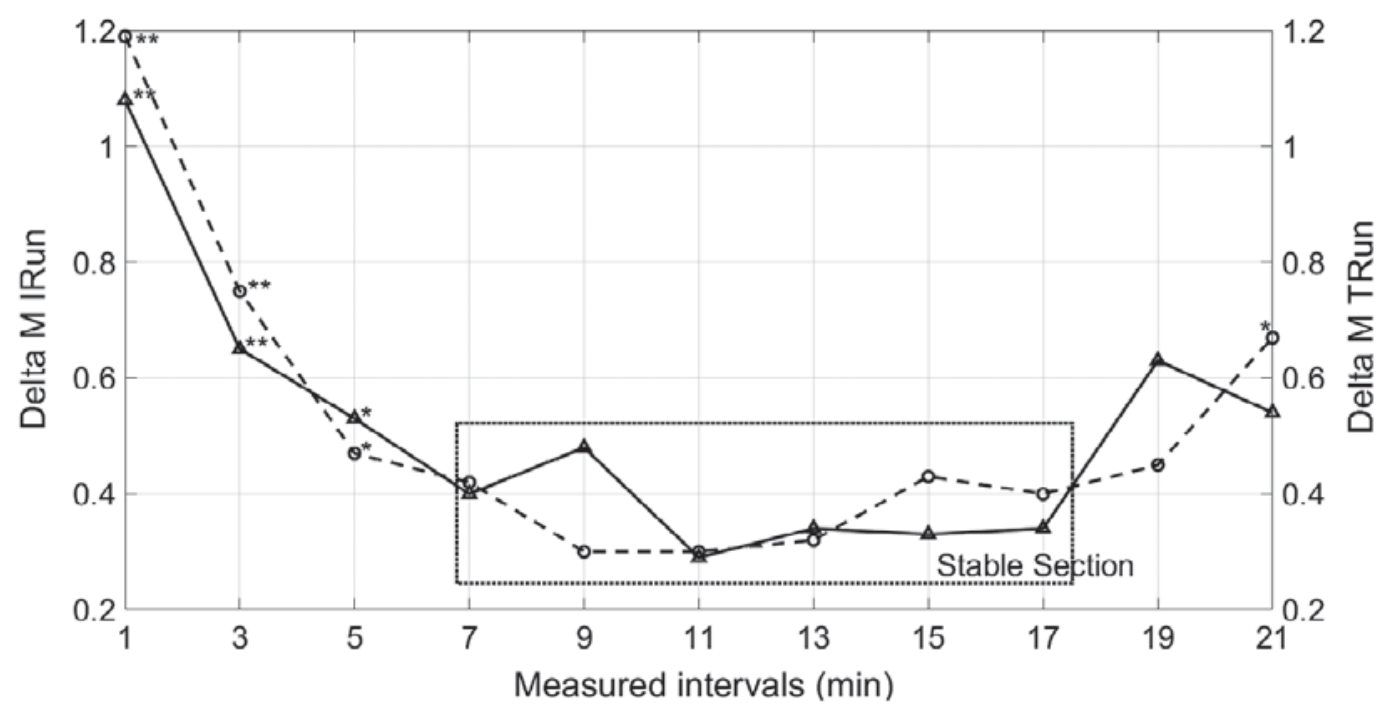

Figure 4. Delta $M$ analysis for IRun (continuous line with triangles) and TRun (dashed line with circles). Notes: Stable section is framed, while ${ }^{* *}$ indicates highly significant differences $(p<0.001),{ }^{*}$ indicates significant differences $(p<0.05)$.

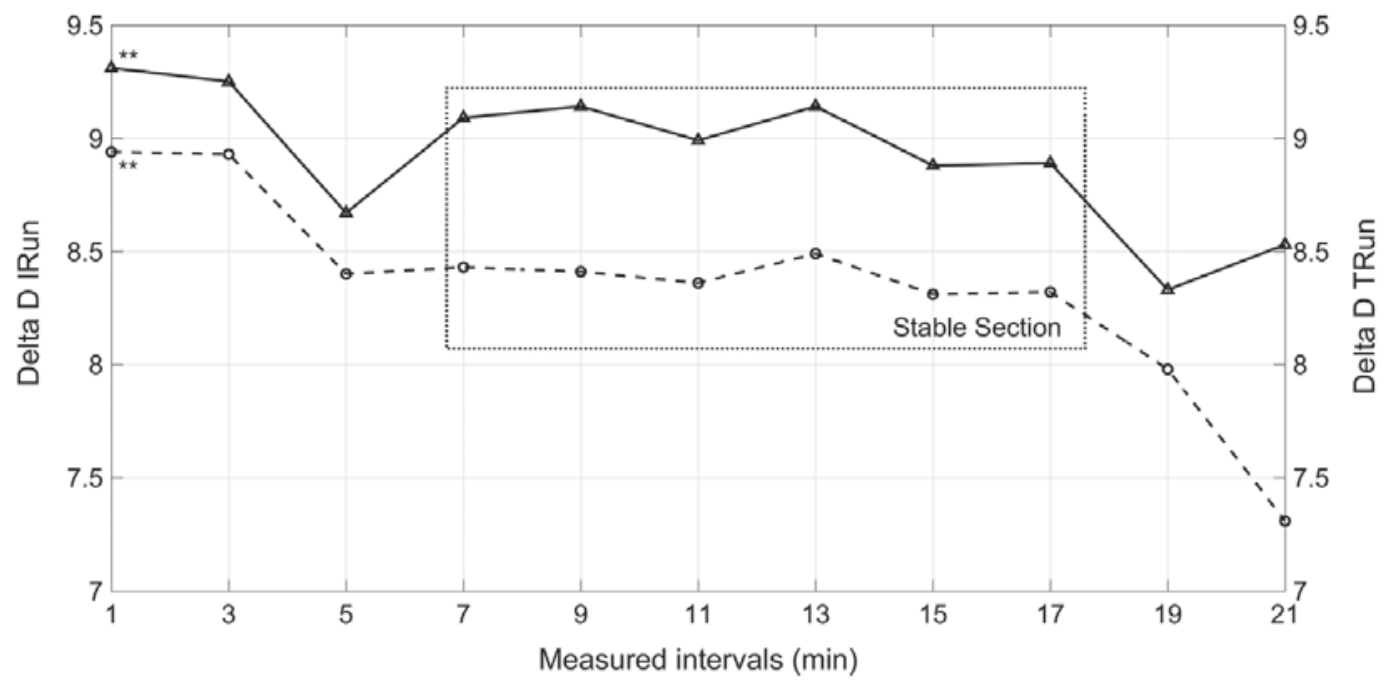

Figure 5. Delta $D$ analysis for IRun (continuous line with triangles) and TRun (dashed line with circles). Notes: Stable section is framed, while ${ }^{* *}$ indicates highly significant differences $(p<0.001),{ }^{*}$ indicates significant differences $(p<0.05)$.

were expected for a triathlon run, it is remarkable that a stand-alone run also produces initially elevated values concerning the individual running characteristics. That raises the question what could have led to this phenomenon?

It is important to note the setting of the IRun: the participants started to warm-up ten minutes before the run, including approximately four minutes of self-selected speed with a few speed-ups, before they had to run six minutes at their final test pace to get used to the velocity. Once the athletes stopped the warm-up, the test supervisor started the sensors and connected them to the computer taking between 10 and $30 \mathrm{~s}$. This gap was probably too long to guarantee a fluent transition and so the neuromuscular system had to renew the running movement, leading to the shown discrepancies. In future, it might be preferable to start the sensors before the warm-up and while a person is cycling prior to the TRun. 
Thus, it is planned to collect the data on a portable and programmed smartphone that will be attached to the athletes' upper arm so that it is no longer necessary to accompany the runners on a bike.

Another potential impact is the difference in physical effort before the actual start of TRun and IRun (Millet, Vleck, \& Bentley, 2009), i.e., 40-min cycling (including the warm-up) and a 10-min warm-up run, respectively. By rating these physical efforts, it is obvious that the preload of the TRun is 30 min longer and thus likely results in a more strenuous effect. It also places demands on other major muscle groups (Bijker, de Groot, \& Hollander, 2002) resulting in a different movement pattern which might have to be renewed afterwards during the run. This renewal of pattern must not be judged negatively, it actually represents the impact during a triathlon race, and this is what the current study attempted to quantify (Bonacci, Blanch, Chapman, \& Vicenzino, 2010; Chapman, Hodges, Briggs, Stapley, \& Vicenzino, 2010; Chapman, Vicenzino, Blanch, Dowlan, \& Hodges, 2008; Chapman et al., 2009). Thus, the first speculative question is if the IRun onset is elevated simply because of the preload, then the TRuns' initial intervals are only higher because of the longer and different influence. Secondly, it is also interesting to find out the magnitude attributed to the triathlon specificity mentioned above. To answer these questions, a so-called run-run test, where the participants were asked to run another 40-min session (10-min warm-up and 30-min preload run) at the same intensity as the bike ride, would ensure that the preload had the same duration. This test should definitely be considered in future research although a 40-min running session as a preload might be too stressful because it is physically much more demanding compared to the cycling session due to the impact of gravity (Munro, Miller, \& Fuglevand, 1987). At that point, it should be considered either to shorten the duration for both conditions or to replace the 40 -min run with a preload run according to a 40-min cycling session. As a third aspect, the triathlon transition study had to deal with critical situations and several problems. Though the American participants had all been measured on an indoor track, it was even harder to collect the German data because often unsettled weather conditions in May and June 2016.

Beyond the mentioned explanations, recent findings (unpublished research) reveal another possible approach dealing with the nature of starting a run or resuming after a short break. During the past months, we analysed 90 healthy persons while walking (below $6 \mathrm{~km} / \mathrm{h}$ ), running (intensity: RPE of 4) and cycling (three different cadences: 60, 80 and $100 \mathrm{rpm}$ ) and were able to reproduce the present results. As described earlier, it is known anecdotally among athletes that you have to find a rhythm just after starting an activity. This process might underlie an initial transient oscillation, as it is known from dynamical systems (Precht, Volt, \& Kraft, 2005). Such systems, like control systems, an electrical network or the complex human body, are characterised by interacting with their environment. If a system was affected by an internal or a nearby perturbation, it takes some time to even out to a balanced condition, an asymptotical equilibrium called attractor. The crossed trajectories are called transients. Once initialised, these transients show rapidly changing and mostly irregular behaviour over a short-lived period until settling down to the steady-state condition (Precht et al., 2005). Hence, this new aspect concerning the running nature may open new possibilities of how to start a run or a race, especially for shorter distances where every second counts. If this influence of neuromuscular adjustments can be demonstrated and replicated in our current and future studies, it would expand the field of enhancing running performance by a new regulating tool. Upcoming work may yield the 
individual extent (gender specific, anthropogenic, performance-related differences, etc.) of the described instances and whether they might be improved by various training methods and tactical variations, like pacing strategies. Pacing includes not only the variation of the race speed (Friel \& Vance, 2013, p. 402; Thompson, 2015, chapter 10), but also aspects like the choice of the most adequate warm-up method (Friel \& Vance, 2013, p. 211), the time window afterwards waiting for the start signal (p. 217) or the role of stops at aid stations especially in long-distance events (Vance, 2016). Moreover, to get back to the main topic of transition runs in duathlon or triathlon events, coaches have already manipulated various strategies during the end of the bike split, or in transition, to improve the final run. The novel knowledge of using the attractor method could support these attempts with objective feedback about individual success. In future, we aim to develop a specific instrument, which is sensitive enough to detect these performance fluctuations at the onset and during runs using our newly developed approach. But then, the general question remains which other factors cause time loss in a triathlon run compared to a single run over the same distance?

At this point, it is important to note that the investigated parameters refer to neuromuscular characteristics of the movement. Physiological and emotional data influencing running performance have been disregarded so far. Previous research and as well our own data shows discrepancies between a triathlon run and a stand-alone run. For example, according to the subjective feeling (Figure 6), the rate of perceived exertion over the 5,000 $\mathrm{m}$ runs between both conditions is considerably higher during the TRun (light grey bars).

Millet and Vleck (2000) reported an increase in oxygen-uptake resulting in a higher cost of running in a transition run compared to a control run. This results in a diminished run off-the-bike performance. Further research groups underlined these findings (Guezennec, Vallier, Bigard, \& Durey, 1996; Hausswirth, Bigard, \& Guezennec, 1997; Hue, Le Gallais, Chollet, Boussana, \& Préfaut, 1998). Hausswirth, Le Meur, Bieuzen, Brisswalter, and Bernard (2010) discussed several other physiological factors, e.g., how redistribution of the blood flow from leg dominance during the cycling to a whole-body distribution during running could have influenced the running performance, particularly at high intensities (p. 1,121). Besides, several other authors published various factors for the increase in energy cost during the transition run, such as glycogen depletion, ventilator muscle fatigue, dehydration or leg muscle fatigue (Guezennec et al., 1996; Hausswirth, Bigard, Berthelot, Thomaidis, \& Guezennec, 1996; Hausswirth et al., 1997; Hue et al., 1998; Millet, Millet, Hofmann, \& Candau, 2000; Millet \& Vleck, 2000).

Another reason for these results in triathlon events could be a changed ankle stiffness (Mueller, Knechtle, Knechtle, \& Toigo, 2015; Rabita, Slawinski, Girard, Bignet, \& Hausswirth, 2011). Reduced stiffness of the muscle-tendon complex caused by the exhaustive cycling preload is known to be 'negatively correlated with contact time and positively correlated with maximal ground reaction force' (Mueller et al., 2015, p. 800).

Finally, we found that minute 21 for the $\delta M$ of the IRun was as well significantly different compared to the SS. At this time, it remains to be discovered finally what caused this finding and why it differs from TRun when the 5,000 $\mathrm{m}$ split was performed in the same way. Possibly, this phenomenon occurred due to emotional reasons; some athletes had already quit the test mentally or in contrast to that accelerated because they knew it was their final lap after a monotonous run (without cycling before). 


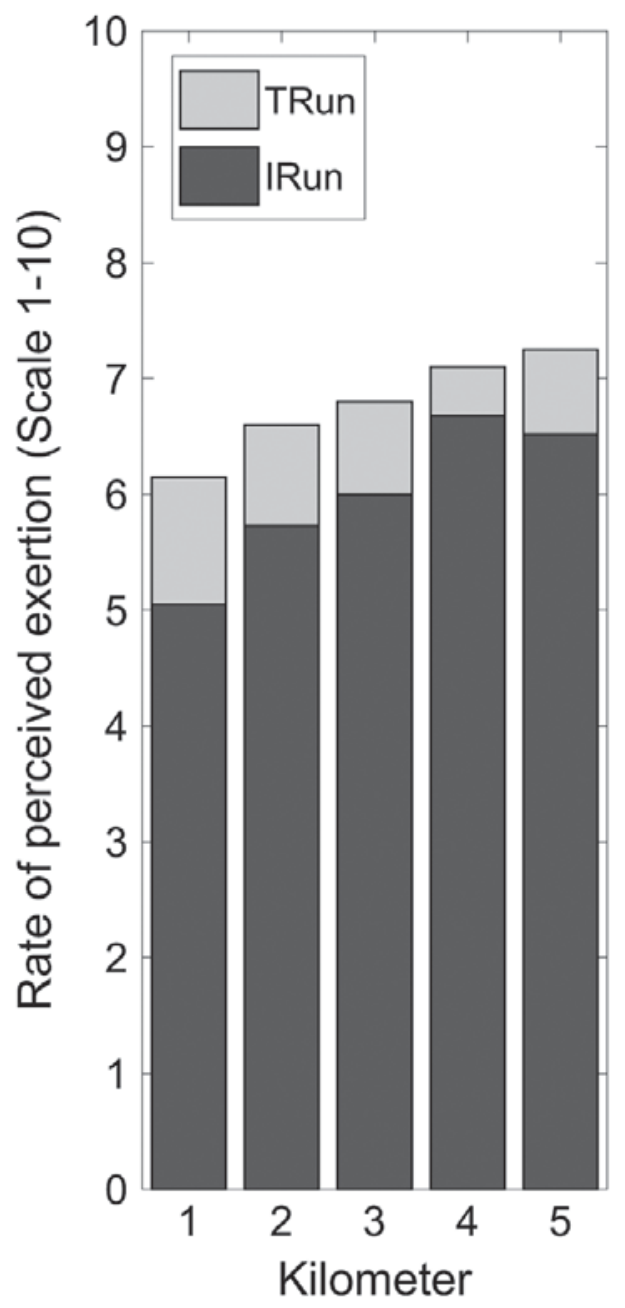

Figure 6. Rate of perceived exertion during IRun and TRun (Scale 1-10). Notes: IRun is shown in dark bars, TRun in light bars.

\section{Conclusion}

The present study dealt with the analysis of a triathlon run in contrast to an isolated running split. Thirty-four athletes were tested performing 5,000 m runs paced in their threshold speed. Finally, independent of the preload with or without cycling, the results show that once a running split has started, it takes some minutes until an athlete found his or her rhythm of an optimal individual running style. These findings were expected for the cycle-run condition, but since the results were similar for the stand-alone run, they cast a completely new light on the nature of running in general. After taking everything into consideration, it is not fully understood why some triathletes run better off the bike than others, but the attractor method has the potential of being another subtle tool to fine-tune athletes' performance. It is sensitive enough to detect subtle changes in individual running behaviour. That provides encouragement to develop an objective measurement instrument to gain more information about the underlying comprehension of human running movement. If this novel view is confirmed in future studies, coaches may have to rethink their pacing strategies to enhance athletes' performance. 


\section{Disclosure statement}

No potential conflict of interest was reported by the authors.

\section{ORCID}

Christian Weich (iD http://orcid.org/0000-0002-3364-043X

\section{References}

Alligood, K., Sauer, T., \& Yorke, J. (1997). Chaos-An introduction to dynamical systems. New York, NY: Springer.

Bijker, K., de Groot, G., \& Hollander, A. (2002). Differences in leg muscle activity during running and cycling in humans. European Journal of Applied Physiology, 87, 556-561.

Bonacci, J., Blanch, P., Chapman, A., \& Vicenzino, B. (2010). Altered movement patterns but not muscle recruitment in moderately trained triathletes during running after cycling. Journal of Sports Sciences, 28, 1477-1487.

Borg, G. (1998). Borg's perceived exertion and pain scales. Champaign: Human Kinetics.

Chapman, A., Hodges, P., Briggs, A., Stapley, P., \& Vicenzino, B. (2010). Neuromuscular control and exercise-related leg pain in triathletes. Medicine and Science in Sports and Exercise, 42, 233-243.

Chapman, A., Vicenzino, B., Blanch, P., Dowlan, S., \& Hodges, P. (2008). Does cycling affect motor coordination of the leg during running in elite triathletes? Journal of Science and Medicine in Sport, 11, 371-380.

Chapman, A., Vicenzino, B., Hodges, P., Blanch, P., Hahn, A., \& Milner, T. (2009). A protocol for measuring the direct effect of cycling on neuromuscular control of running in triathletes. Journal of Sports Science, 27, 767-782.

Coen, B., Urhausen, A., \& Kindermann, W. (2001). Individual anaerobic threshold: Methodological aspects of its assessment in running. International Journal of Sports Medicine, 22, 8-16.

Friel, J., \& Vance, J. (2013). Triathlon science-The ultimate nexus of knowledge and performance. Champaign: Human Kinetics.

Gohlitz, D., Große, S., \& Witt, M. (1994). Darstellungen von veränderungen der schrittlänge und schrittfrequenz beim übergang vom radfahren zum laufen zur kennzeichnung der dauer von übergangsphasen im duathlon (pilotuntersuchung). [Representation of changes of step length and step frequency during transition from cycling to running for identification of the duration of transitional periods in duathlon (pilot study)]. In M. Engelhardt, B. Franz, G. Neumann, \& A. Pfützner (Ed.), Triathlon: Medizinische und methodische Probleme des Trainings. Internationales Triathlon-Symposium Bad Endorf (Vol. 9, pp.131-135). Hamburg: Czwalina.

Guezennec, C., Vallier, J., Bigard, A., \& Durey, A. (1996). Increase in energy cost of running at the end of a triathlon. European Journal of Applied Physiology and Occupational Physiology, 73, 440-445.

Hausswirth, C., Bigard, A., Berthelot, M., Thomaidis, M., \& Guezennec, C. (1996). Variability in energy cost of running at the end of a triathlon and a marathon. International Journal of Sports Medicine, 17, 572-579.

Hausswirth, C., Bigard, A., \& Guezennec, C. (1997). Relationships between running mechanics and energy cost of running at the end of a triathlon and a marathon. International Journal of Sports Medicine, 18, 330-339. 
Hausswirth, C., Le Meur, Y., Bieuzen, F., Brisswalter, J., \& Bernard, T. (2010). Pacing strategy during the initial phase of the run in triathlon: Influence on overall performance. European Journal of Applied Physiology, 108, 1115-1123.

Hue, O., Le Gallais, D., Chollet, D., Boussana, A., \& Préfaut, C. (1998). The influence of prior cycling on biomechanical and cardiorespiratory response profiles during running in triathletes. European Journal of Applied Physiology and Occupational Physiology, 77, 98-105.

Lyapunov, A. (1992). The general problem of the stability of motion. Washington, DC: Taylor \& Francis.

Millet, G., Millet, G., Hofmann, M., \& Candau, R. (2000). Alterations in running economy and mechanics after maximal cycling in triathletes: Influence of performance level. International Journal of Sports Medicine, 21, 127-132.

Millet, G., Vleck, V., \& Bentley, D. (2009). Physiological differences between cycling and running: Lessons from triathletes. Sports Medicine, 39, 179-206.

Millet, G., \& Vleck, V. (2000). Physiological and biomechanical adaptions to the cycle to run transition in Olympic triathlon: Review and practical recommendations for training. British Journal of Sports Medicine, 34, 384-390.

Mueller, S., Knechtle, P., Knechtle, B., \& Toigo, M. (2015). An ironman triathlon reduces neuromuscular performance due to impaired force transmission and reduced leg stiffness. European Journal of Applied Physiology, 115, 795-802.

Munro, C., Miller, D., \& Fuglevand, A. (1987). Ground reaction forces in running: A reexamination. Journal of Biomechanics, 20, 147-155.

Perc, M. (2005). The dynamics of human gait. European Journal of Physics, 26, 525-534.

Precht, M., Volt, K., \& Kraft, R. (2005). Mathematik 2 für Nichtmathematiker [Mathematics 2 for non-mathematicians]. Muenchen: Oldenbourg Wissenschaftsverlag.

Rabita, G., Slawinski, J., Girard, O., Bignet, F., \& Hausswirth, C. (2011). Spring-mass behaviour during exhaustive run at constant velocity in elite triathletes. Medicine and Science in Sports and Exercise, 43, 1157-1167.

Shannon, C. (1948). A mathematical theory of communication. Bell System Technical Journal, 27, 623-656.

Shannon, C. (1949). Communication in the presence of noise. Proceedings of the Institute of Radio Engineers, 37, 10-21.

Stafford, I. (2012, August 4). Disappointment for Jenkins as Spirig wins dramatic photo finish in triathlon. Retrieved from http://www.dailymail.co.uk/sport/olympics/article-2183568/London2012-Olympics-Nicola-Spirig-wins-triathlon-photo-finish.html\#ixzz4Dnd5kEuD

Stegmann, H., Kindermann, W., \& Schnabel, A. (1981). Lactate kinetics and individual anaerobic threshold. International Journal of Sports Medicine, 2, 160-165.

Sehle, A., Vieten, M., Sailer, S., Muendermann, A., \& Dettmers, C. (2014). Objective assessment of motor fatigue in multiple sclerosis: The Fatigue index Kliniken Schmieder (FKS). Journal of Neurology, 261, 1752-1762.

Taken, F. (1981). Detecting strange attractor in turbulence. In D. Rand \& L. Young (Eds.), Dynamical systems and turbulence (pp. 366-381). New York, NY: Springer.

Thomas, D., Reading, J., \& Shepard, R. (1992). Revision of the physical activity readiness questionnaire (PAR-Q). Canadian Journal of Sports Science, 17, 338-345.

Thompson, K. (2015). Pacing: Mit der richtigen Strategie optimale Leistungen erbringen [Pacing: Individual strategies for optimal performance]. Hamburg: spomedis.

Vance, J. (2016). Triathlon 2.0-Data-driven performance training (Chapter 12). Champaign: Human Kinetics.

Vieten, M., \& Jensen, R. L. (2015). The attractor method-A technique to quantify differences of cyclic processes and their variability. In F. Colloud, M. Domalain, \& T. Monnet (Ed.), Proceedings of XXXII Congress of the International Society of Biomechanics in Sports (pp. 511-514). University of Poitiers, France.

Vieten, M., Sehle, A., \& Jensen, R. L. (2013). A novel approach to quantify time series differences of gait data using attractor attributes. PLoS One, 8, 1-10.

Vleck, V., Burgi, A., \& Bentley, D. (2006). The consequences of swim, cycle, and run performance on overall result in elite Olympic distance triathlon. International Journal of Sports Medicine, 27, 43-48. 
Walsh, M., Hohl, J., Strang, A., \& Haworth, J. (2014). Specificity: Brick training for the bike to run transition in triathlon. In R. Jensen, W. Ebben, E. Petushek, C. Richter, \& K. Roemer (Ed.), Proceedings of the XXVIII Conference of the International Society of Biomechanics in Sports (Vol. 28, pp. 761-764). East Tennessee State University, USA.

Walsh, J., Stamenkovic, A., Lepers, R., Peoples, G., \& Stapley, P. (2015). Neuromuscular and physiological variables evolve independently when running immediately after cycling. Journal of Electromyography and Kinesiology, 25, 887-893.

Wellington, C. (2013). A life without limits (p. 7). London: Constable.

Witt, M. (1994). Biomechanische Untersuchungen zum Belastungswechsel im Triathlon [Biomechanical studies concerning load changes in triathlon]. In M. Engelhardt, B. Franz, G. Neumann, \& A. Pfützner (Eds.), Triathlon-Medizinische und methodische Probleme des Trainings (pp. 137-144). Hamburg: Czwalina. 